\title{
Luis Toharia \& Inmaculada Cebrián, La temporalidad en el empleo : atropmiento y trayectorias
}

Joaquín Pitarch

\section{(2) OpenEdition}

\section{Journals}

Édition électronique

URL : http://journals.openedition.org/travailemploi/4320

DOI : 10.4000/travailemploi.4320

ISSN : 1775-416X

Éditeur

DARES - Ministère du Travail

\section{Édition imprimée}

Date de publication : 15 septembre 2008

Pagination : 117-119

ISSN : 0224-4365

\section{Référence électronique}

Joaquín Pitarch, « Luis Toharia \& Inmaculada Cebrián, La temporalidad en el empleo : atropmiento y trayectorias », Travail et Emploi [En ligne], 115 | juillet-septembre 2008, mis en ligne le 18 février 2011 consulté le 22 septembre 2020. URL : http://journals.openedition.org/travailemploi/4320 ; DOI : https://doi.org/10.4000/travailemploi.4320 


\section{El problema de la temporalidad en España: un diagnóstico}

(Le problème de l'emploi temporaire en Espagne: diagnostic)

Luis Toharia (dir.), Jesús Cruz, Cecilia Albert, Carlos García Serrano, Javier Calvo, Inmaculada Cebrián, Virginia Hernanz, Miguel Angel Malo et Gloria Moreno.

\author{
Madrid, Ministère du Travail et des Affaires \\ Sociales, collection Économie et Sociologie \\ du Travail, 2005.
}

\section{La temporalidad en el empleo: atropamiento y trayectorias}

\author{
(L'emploi temporaire: catégories et \\ trajectoires) \\ Luis Toharia et Inmaculada Cebrián
}

\author{
Madrid, ministère du Travail et des Affaires \\ sociales, 2007
}

\section{Lu par Joaquín Pitarch (université d'Alcalá de Henares)}

Le marché du travail espagnol continue à souffrir de graves problèmes que les réformes du travail menées depuis 1997 n’ont pas permis de résoudre. Parmi ces problèmes, on note un taux d'activité des femmes relativement faible, la persistance d'un taux de chômage élevé, bien qu'identique à la moyenne des vingt-cinq États-membres de l'Union européenne et, surtout, un taux élevé de contrats temporaires.

Suite à la rupture du principe de causalité, conformément au décret royal portant loi 1989-1984 relatif à l'utilisation du contrat temporaire no causal « sans raison claire et précise » comme mesure pour favoriser l'emploi, le recours au contrat à durée déterminée par les entreprises a connu une augmentation. Ce changement a permis de rendre plus flexible le marché du travail espagnol et d'améliorer l'adaptation des entreprises aux changements économiques sans avoir à supporter les coûts élevés des licenciements des travailleurs sous contrat à durée indéterminée. C’est pourquoi, et malgré les efforts réalisés jusqu'à présent dans le but de réduire la proportion de contrats temporaires et d'encourager le recours aux contrats à durée indéterminée, le marché du travail espagnol connaît un taux de contrats temporaires élevé, supérieur à celui de ses pays voisins, représentant plus de $30 \%$.

L'ouvrage intitulé El problema de la temporalidad en España: Un diagnóstico, réalisé sous la direction de Luis Toharia, présente une analyse de la situation de l'emploi en Espagne au cours des vingt-cinq dernières années et, plus précisément, des contrats temporaires, thème qui a donné lieu à divers débats sur la précarité de l'emploi en résultant, sur la volatilité de l'emploi, sur les freins à la formation, etc.

L'ouvrage comprend six chapitres. Le premier chapitre propose une analyse du modèle de contrat temporaire espagnol, caractérisé par ce qu'il est convenu d'appeler la marge de flexibilité. Afin de mener à bien cette étude, les données-clés du travail sous contrat temporaire en Espagne, ainsi qu'une étude normative du sujet, sont exposées. L'étude du droit du travail permet de mieux comprendre l'évolution de la part des emplois temporaires au cours des vingt-cinq dernières années.

Le second chapitre offre une analyse des modèles de recours aux contrats temporaires des pays voisins montrant au final que le modèle espagnol présente un taux d'emplois temporaires qui n'a d'équivalent dans aucun des pays étudiés. Afin d'expliquer ce fait, les auteurs ont analysé le droit du travail propre à chaque pays et ont noté que l'Espagne conserve un nombre significatif de similitudes avec certains pays, comme la France ou l'Italie, non seulement dans le domaine de la réglementation des contrats temporaires, mais également en ce qui concerne les différences entre les coûts de licenciement des travailleurs sous contrat à durée indéterminée et ceux des travailleurs sous contrat temporaire. Cela étant, mises à part les similitudes en matière de réglementation, ces trois pays affichent des taux d'emploi temporaire fortement différents.

Cependant, ces similitudes en matière de réglementation n'ont pas toujours existé. En effet, les différences s'expliquent par l'utilisation pernicieuse des contrats temporaires par les chefs d'entreprise, suite à la réforme de 1984 et jusqu'en 1994, année à partir de laquelle ont été fixées des limites à l'utilisation des contrats temporaires comme mesure permettant de favoriser uniquement l'emploi de certaines catégories de personnes. Les auteurs ont également analysé les caractéristiques personnelles des travailleurs ainsi que les structures de production de chaque pays, mais ces éléments permettent tout juste d'expliquer une part infime du taux d'emploi temporaire.

Le troisième chapitre propose une analyse théorique visant à expliquer les causes du travail sous contrat temporaire en Espagne et la persistance de ce dernier malgré les mesures prises lors de la réforme de 1997, telles que celles ayant trait au recours aux contrats à durée indéterminée afin de favoriser l'emploi et les mesures d'incitation économique visant à encourager le recours aux contrats à durée indéterminée. L’analyse conclut que l'emploi temporaire obéit à des causes clairement économiques. Ainsi, les entreprises, dans leur quête de maximisation des bénéfices, décident du niveau optimal de recours aux contrats temporaires permettant de générer un bénéfice maximal. Ce niveau optimal dépendra donc respectivement des coûts de licen- 
ciement et des coûts de rotation du personnel sous contrat à durée indéterminée et du personnel sous contrat temporaire.

Le quatrième chapitre s'attèle à l'analyse des coûts sociaux liés à l'emploi temporaire, coûts non considérés par les entreprises lorsque cellesci établissent le niveau optimal de main-d'œuvre temporaire. Ce chapitre traite plus précisément du rapport entre l'emploi temporaire et les variables suivantes: la formation au sein de l'entreprise, les accidents du travail, les conditions de travail et les dépenses en matière d'assurance chômage. Les estimations économétriques réalisées font apparaître d'importantes différences selon qu'on se place du côté des travailleurs sous contrat temporaire ou du côté des travailleurs sous contrat à durée indéterminée. Les travailleurs sous contrat temporaire ont moins de chances de bénéficier d'une formation, connaissent davantage d'accidents du travail et ont des conditions de travail moins favorables. Toutefois, si l'on considère à la fois la variable relative à l'emploi temporaire et d'autres variables relatives au poste de travail, y compris l'ancienneté, les différences ont tendance à disparaître.

Enfin, le cinquième chapitre évalue les conséquences de la réforme du travail de 1997 quant à l'évolution de l'emploi temporaire. Les données font clairement apparaître l'existence d'un processus de substitution entre les différents types de contrats à durée indéterminée et le fait que les chefs d'entreprises y ont recours de manière stratégique afin de profiter au maximum des conditions économiques du moment. Par ailleurs, les auteurs démontrent que les contrats aidés et ceux transformant les contrats temporaires en contrats à durée indéterminée sont plus instables que les contrats à durée indéterminée classiques, ce qui signifie que les deux premiers types de contrats visent à couvrir les postes de travail les plus instables ainsi que les périodes d'essai des travailleurs; leurs caractéristiques s'apparentent donc davantage à celles des contrats temporaires.

Cet ouvrage ne traite pas en détail du problème des travailleurs enchaînant les contrats temporaires au cours de leur vie professionnelle. Ce thème est en revanche abordé par l'ouvrage de Luis Toharia et Inmaculada Cebrián, intitulé $\mathbf{L} \boldsymbol{a}$ temporalidad en el empleo: atrapamiento y trayectorias, dans lequel sont analysées les catégories de personnes éprouvant les plus grandes difficultés à sortir de l'emploi temporaire et donc incapables d'atteindre une certaine stabilité d'emploi.

L'objet de l'étude était de comparer les parcours professionnels des travailleurs sous contrat temporaire et ceux des travailleurs sous contrat à durée indéterminée grâce à une nouvelle source de données statistiques, La Muestra Continua de Vidas Laborales (MCVL) (échantillon permanent de vies professionnelles). Cet échantillon permet d'analyser le marché du travail espagnol d'une manière différente à celle utilisée dans l'ouvrage commenté précédemment.
La MCVL permet d'analyser les emplois sur des périodes plus longues mais offre surtout la possibilité de réaliser un suivi longitudinal des parcours individuels. L'ouvrage se compose de six chapitres principaux faisant suite à un premier chapitre introductif de présentation de la base de données et des restrictions de champ effectués afin d'obtenir le sous-échantillon qui fait l'objet de l'étude.

Les chapitres deux et trois analysent les caractéristiques fondamentales telles que l'âge et le nombre de périodes de cotisation des différentes cohortes de cotisants ainsi que le parcours des travailleurs sous contrat temporaire et de ceux sous contrat à durée indéterminée au cours de la période allant de 1980 à 2004. Les résultats obtenus font apparaître que les travailleurs sous contrat temporaire sont plus jeunes que les travailleurs sous contrat à durée indéterminée. D’après la MCVL, près de $90 \%$ des travailleurs de moins de 25 ans n'ont connu que des contrats temporaires en 2004, cette proportion diminuant avec l'âge. L'analyse des catégories de personnes en fonction de la première année de cotisation au régime de Sécurité sociale montre que le nombre de personnes concernées varie en fonction de l'évolution du marché du travail. Ainsi, en période de crise économique, le nombre de nouveaux cotisants diminue alors qu'en période de croissance, leur nombre augmente. En 2004, cette analyse fait ressortir un autre résultat notable: les personnes plus âgées connaissent moins de contrats temporaires que les plus jeunes. Par ailleurs, l'âge d'accès au premier véritable emploi, à savoir le premier emploi à temps complet d'une durée supérieure à trois mois, est également estimé. Les résultats obtenus montrent un décalage entre l'âge de début de cotisation au régime de Sécurité sociale et l'âge d'accès à un véritable emploi d'environ deux ans. Ce décalage est plus élevé dans le cas des travailleurs sous contrat temporaire.

Concernant le nombre de périodes de cotisation au régime de Sécurité sociale, l'échantillon indique que la moyenne est de quatorze périodes. Dans le cas des travailleurs n'ayant conclu que des contrats temporaires au cours de l'année 2004, la moyenne du nombre de ces périodes est de dix-sept, et monte à trente-deux dans le cas des travailleurs les plus âgés. Dans le même temps, les travailleurs n’ayant conclu que des contrats à durée indéterminée en 2004 affichent une moyenne de dix périodes, quatorze dans le cas des travailleurs les plus âgés. Les résultats font également apparaître que le nombre de périodes de cotisation des hommes ayant conclu des contrats temporaires ou à la fois des contrats temporaires et des contrats à durée indéterminée en 2004 est supérieur à celui des femmes. Il est important de signaler que le contrat temporaire constitue de plus en plus fréquemment une passerelle d'entrée vers le marché du travail. Ainsi, deux travailleurs sur trois ayant conclu des contrats à durée indéterminée au cours de l'année 2004 avaient précédemment connu 
un contrat temporaire. Cette proportion diminue si l'on prend en compte la date d'entrée sur le marché du travail; on observe l'effet inverse avec l'âge. Par ailleurs, trois travailleurs sur quatre ayant uniquement conclu des contrats temporaires en 2004 n'ont jamais conclu de contrat à durée indéterminée.

Enfin, le chapitre trois propose une analyse multivariée de la probabilité d'être sous contrat à durée indéterminée sans avoir connu de contrat temporaire en 2004 et de celle d'être sous contrat temporaire sans jamais avoir connu de contrat à durée indéterminée au cours de la même année. L'analyse fait apparaître des différences significatives entre les hommes et les femmes vis-à-vis des contrats temporaires; les femmes demeurent en situation de précarité (travail temporaire) et elles ont moins accès aux contrats à durée indéterminée. L’âge et l'expérience jouent également un rôle décisif pour la stabilité professionnelle.

Le chapitre quatre examine la durée des périodes de cotisation, à la fois pendant les périodes d'emploi et pendant les périodes de perception des allocations chômage. On note que la durée totale des périodes de cotisation augmente au fil du temps. De même, si l'on prend en compte la durée relative des périodes de cotisation, il apparaît que les cotisations totales des travailleurs n'ayant connu que des contrats à durée indéterminée au cours de l'année 2004 reposent sur $88 \%$ du temps de travail maximal possible sur une année, $84 \%$ de ce temps correspondant aux périodes d'emploi et les $4 \%$ restants aux périodes de perception des allocations chômage. Cependant, dans le cas des travailleurs n'ayant connu que des contrats temporaires en 2004, les cotisations maximales totales reposent sur $65 \%$ du temps de travail maximal possible, $58 \%$ de ce temps correspondant aux périodes d'emploi et le reste aux périodes de chômage.

Le chapitre cinq traite, quant à lui, des différences entre les bases de cotisation. En analysant l'année 2004, les auteurs concluent que les bases de cotisation, dans le cas des travailleurs n'ayant connu que des contrats à durée indéterminée, sont supérieures à celles des autres catégories prises en compte. Cette analyse montre notamment que la base de cotisation des femmes est inférieure à celle des hommes et que les travailleurs n'ayant signé que des contrats à durée indéterminée au cours de l'année 2004, n'étant pas précédemment des contrats temporaires, ont un avantage par rapport aux autres catégories de personnes.

Concernant l'étude portant sur l'expérience professionnelle des travailleurs ayant cotisé en tant que salariés du 23 mars 2003 au 23 septembre 2005, le chapitre six révèle que le temps de travail total sur la base duquel sont calculées les cotisations s'approche, en moyenne, des trois quarts du temps total de travail potentiel. Dans le cas des travailleurs n'ayant signé que des contrats temporaires au cours de l'année 2004, on note que la durée moyenne de la période de cotisation est d'environ dix-huit mois. Cette analyse a permis d'estimer le nombre de travailleurs qui, à la fin de la période d'observation, se trouvent sous contrat temporaire, ont travaillé plus de vingt-quatre mois et ont connu au moins deux contrats à durée déterminée au sein de la même entreprise; leur nombre se situe aux alentours de 500000 . Ce chiffre prend en compte la limite maximale de transformation automatique des contrats temporaires en contrats à durée indéterminée conformément à la réforme du travail de juin 2006. Cependant, l'analyse a également fait apparaître une autre catégorie de travailleurs, plus importante en nombre, non prise en compte dans le cadre de cette réforme du travail, à savoir les personnes ayant travaillé plus de vingt-quatre mois au cours des trente derniers mois au sein de la même entreprise sous un seul et même contrat temporaire; on estime que cette catégorie de travailleurs est susceptible de représenter plus de 600000 personnes.

Enfin, le chapitre sept porte sur l'étude des travailleurs entrés sur le marché du travail en 2004. Cette catégorie se compose de jeunes de moins de 35 ans. La majeure partie de ces travailleurs est entrée sur le marché du travail par le biais d'un contrat temporaire ( $87 \%$ ) et à peine plus de la moitié d'entre eux cotise toujours au régime de Sécurité sociale au 23 septembre 2005. Les travailleurs ayant accédé au régime de Sécurité sociale via un contrat à durée indéterminée représentent quant à eux un peu moins de $13 \%$, dont $93 \%$ n’ayant connu qu'un contrat à durée indéterminée et $3 \%$ ne faisant plus partie du régime de Sécurité sociale. Cependant, au 23 septembre 2005, 80\% des travailleurs ayant accédé au régime de Sécurité sociale en 2004 en faisaient toujours partie. Ce pourcentage est plus important dans le cas des travailleurs arrivés sur le marché du travail via un contrat temporaire transformé que dans celui des travailleurs arrivés sur le marché du travail via un contrat à durée indéterminée classique ou via un contrat à durée indéterminée permettant de favoriser l'emploi. Ainsi, bien que les personnes arrivant sur le marché du travail via un contrat à durée indéterminée soient beaucoup moins nombreuses, elles ont davantage de chances de conserver leur emploi.

En conclusion, le marché du travail espagnol se caractérise par un fort taux de contrats temporaires, problème que les réformes du travail menées depuis 1997 ne sont pas parvenues à résoudre. Les différentes études réalisées ont tenté d’analyser les catégories de personnes principalement touchées par le recours aux contrats temporaires, le parcours professionnel de ces personnes ainsi que les caractéristiques individuelles des travailleurs se trouvant enfermés dans le cycle du travail temporaire. Pour y parvenir, une nouvelle source de statistiques appelée Muestra Continua de Vidas Laborales (échantillon permanent de vies professionnelles) a été utilisée, cette source permettant d'analyser le travail temporaire sous un angle nouveau. 